\title{
Estimation and Removal of T Wave Component in Atrial Flutter ECG to Aid Non-Invasive Localization of Ectopic Source
}

\author{
Muhammad Haziq Kamarul Azman ${ }^{1}$, Olivier Meste ${ }^{1}$, Kushsairy Kadir ${ }^{2}$, Decebal Gabriel Latcu ${ }^{3}$ \\ ${ }^{1}$ Université Côte d'Azur, CNRS, I3S, France \\ ${ }^{2}$ Universiti Kuala Lumpur, Malaysia \\ ${ }^{3}$ Cardiology Department, Centre Hospitalier Princesse Grace, Monaco
}

\begin{abstract}
The use of the ECG as a diagnostic tool for analyzing supraventricular arrhythmia avoids invasive procedures prior to clinical intervention. Analysis of atrial activity may indicate properties of the underlying arrhythmia. In atrial flutter, there is a high synchroneity between atrial and ventricular activity. Statistical and classical separation methods are unlikely to work due to this dependency, impeding the extraction of the atrial activity. We propose a method using least square polynomial estimation to model the $T$ wave component and subtract it from overlapped $f$ waves. After pre-processing, an initial $f$-wave was carefully segmented, representing pure atrial activity. Subsequent $f$-waves were detected and corrected by subtracting the $T$ wave, modeled as a sum of weighted polynomials which minimize the least square error. To evaluate the method, vectorcardiographic loops were obtained using the inverse Dower transform. Loop parameters were obtained from eigenvectors and eigenvalues issued from principal component analysis. Using degree 3 polynomials allowed recovery of overlapped $f$-waves with signifcant improvement in 3 of 4 parameters $(p<0.05)$. $f$-waves overlapped by $T$ waves can thus successfully be recovered using this method.
\end{abstract}

\section{Introduction}

Supraventricular arrhythmia (SVA) is a cardiac disorder whereby the normal activation rhythm of the atria is disrupted. The natural cardiac pacemaker is suppressed by a rapidly activated ectopic cellular source (in the form of a focus or a rotating circuit). Two well-known SVAs are atrial fibrillation (AF), affecting $0.5 \%$ of the worldwide population and atrial tachycardia, often referred to as atrial flutter (AFL). SVAs can be treated using radiofrequency catheter ablation: a technique whereby atrial muscles are destroyed using high-frequency radio waves delivered via an ablation catheter. Upon destruction of pathologic mus- cles, the atrial rhythm returns to normal.

Analysis and evaluation of SVA can be performed using electrocardiographic (ECG) signals. Traditionally, analysis of these ECG traces are done by specialists as a part of a standard procedure prior to intervention. The noninvasive, standard nature of the ECG signal presents a strong benefit for practitioners as it foregoes the need for pre-interventional operations and is universally comparable.

In many cases of SVA, the location of the ectopic source influences the overall difficulty of catheterization. Traditionally, this may only be known once acquisition catheters are placed inside the coronary sinus. Prior knowledge as to which atrium the circuit or focus is located will thus aid in the planning phase, effectively reducing operation time.

Evaluation of the continuous, abnormal atrial activity (AA) may give information on f-wave variability, which was shown to be linked to circuit localization [1]. It had also been shown that careful treatment of these signals and application of signal processing methods may allow analysis and evaluation of cardiac pathologies [2]. However, due to the continuous, rapid nature of atrial depolarisation and variable block ratio, ventricular activity (VA; QRS complex and $\mathrm{T}$ waves) overlaps atrial activity.

Techniques for separating these two sources exist, but relies on particular properties such as source independence or distinction in spectral occupation. In AFL, the continuous and orderly depolarisation of atrial structure results in synchroneity between VA and AA, creating a dependency link. This invalidates classic beat-averaging and source separation methods, which were more suited for AF. Furthermore, f-waves occupy the same spectral band as $\mathrm{T}$ waves, making classical filtering approach useless.

We present an approach that attempts to model the VA using a least square polynomial estimation (LSPE) technique and recover $\mathrm{f}$-waves that are superposed on $\mathrm{T}$ waves. To assess its performance, parameters of vectorcardiographic (VCG) loops derived from these f-waves are evaluated. 


\section{Materials and Method}

\subsection{Data description and pre-processing}

25 recordings of 12-leads standard ECG from the cardiology department at the Centre Hospitalier Princesse Grace in Monaco were acquired from 23 patients in whom AFL occurred. The signals were acquired during ablation procedures using an acquisition system (Bard, USA). All signals were sampled at $2000 \mathrm{~Hz}$ and ranges from 12.04 seconds to 1 minute in length. Sustained episodes of AFL were present during the entire recording.

All signals were filtered between 1 and $100 \mathrm{~Hz}$ with an order 20 and order 3 type II Chebyshev low-pass and highpass filter in order to reject physiological motion noise but to also preserve $\mathrm{f}$-wave onsets. A $50 \mathrm{~Hz}$ finite-impulse response notch filter was used to filter out powerline noise. The signals were then downsampled to $250 \mathrm{~Hz}$ to reduce the number of points.

\subsection{Detection and segmentation of f-wave}

An initial f-wave vector $\mathbf{f}$ of length $N$, spanning from onset to end, was arbitrarily selected from a lead prior to a QRS complex, during a period of long diastole or high AV block ratio (3:1 and above), although with careful consideration on the onsets and ends, f-waves in a 2:1 block ratio setting were also used. It best represents AA free from any VA. This initial f-wave was used to detect subsequent $\mathrm{f}$-waves across the signal.

A sliding window on the original signal is defined as

$$
\mathbf{x}(\tau)=\left[\begin{array}{llll}
x(0+\tau) & x(1+\tau) & \cdots & x(N-1+\tau)
\end{array}\right]^{\mathrm{T}}
$$

where $x(t)$ is the original signal at time $t \in[0 ; M-1]$ and $\tau$ is comprised between $[0 ; M-N+1]$. Both the initial wave and the sliding window were centered by subtracting their respective means before calculating the least square error function

$$
I(\tau)=\|\mathbf{f}-\mathbf{x}(\tau)\|^{2}
$$

The resulting error $I$ presents a zero when $\mathbf{x}\left(\tau_{0}\right)=\mathbf{f}$ and a local minima at $\mathbf{x}\left(\tau_{0}+n T\right), n \in \mathbb{Z}$, where $T$ indicates the f-wave period, due to beat-to-beat variations. Elsewhere, the error is expected to be larger than zero (Fig 1). The minima of $I$ allow us to pre-segment the waves by giving their estimate position in time. The wave onsets were taken at these points and later manually synchronized. f-waves are rejected if they are too close to the preceding end or succeeding onset of a QRS complex.

It was noted during this research that a detection scheme may be made using a generalized likelihood ratio test, which may improve detection performance. This problem

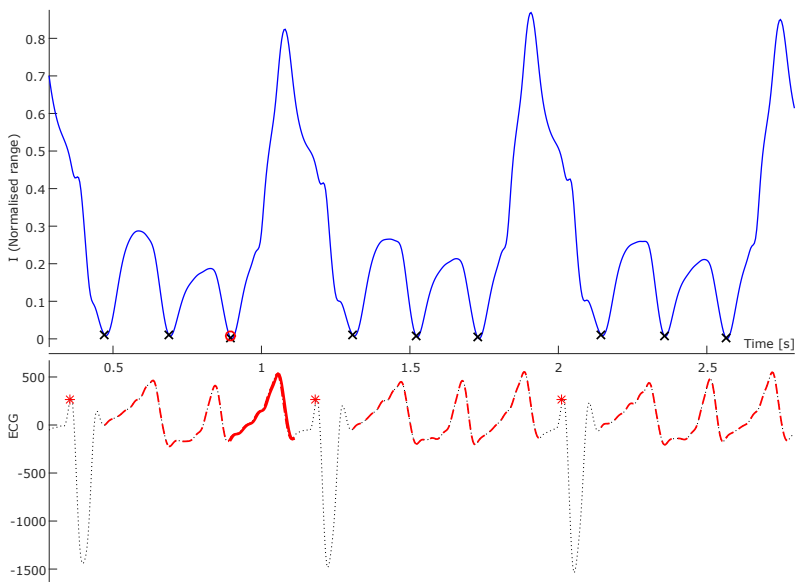

Figure 1. The error function $I$ (top) for recording \#9 (lead V1, bottom). The minima, marked by black crosses, clearly marks the onset of an f-wave on the ECG (dashed red lines). The red circle (where $I=0$ ) and red bolded wave corresponds to the initial reference.

is the subject of a future research and is not treated in this paper.

Pure f-waves (ones that are free from VA) were defined as the f-waves closest to the next QRS complex (indexed using Pan-Tompkins algorithm). In some cases of exceptionally large block ratio, the second last f-wave was also taken. All the isolated $\mathrm{f}$-waves were separated into two sets: $\mathcal{F}_{p}$ and $\mathcal{F}_{o}$, representing pure and overlapped fwaves.

\subsection{Recovery of f-waves using LSPE}

The wave superposition model can be equated to the sum of an $\mathbf{f}$-wave observation $\mathbf{f}_{i}$ and a $\mathrm{T}$ wave model approximated as the sum of polynomials of orders 0 to $K$ weighted by coefficients $b_{i}$

$$
\begin{aligned}
\mathbf{f}_{o, i} & =\mathbf{f}_{i}+\sum_{k=0}^{K} b_{i}(k) \mathbf{m}^{k}=\mathbf{f}_{i}+\mathbf{M} \mathbf{b}_{i} \\
\mathbf{m}^{k} & =\left[\begin{array}{lllll}
0^{k} & 1^{k} & 2^{k} & \cdots & (N-1)^{k}
\end{array}\right]^{\mathrm{T}} \\
\mathbf{M} & =\left[\begin{array}{lllll}
\mathbf{m}^{0} & \mathbf{m}^{1} & \mathbf{m}^{2} & \cdots & \mathbf{m}^{K}
\end{array}\right] \\
\mathbf{b}_{i} & =\left[\begin{array}{lllll}
b_{i}(0) & b_{i}(1) & b_{i}(2) & \cdots & b_{i}(K)
\end{array}\right]^{\mathrm{T}}
\end{aligned}
$$

Thus, we obtain $\mathbf{f}_{i}=\mathbf{f}_{o, i}-\mathbf{M} \mathbf{b}_{i}$. The expression can be inserted in (1) in place of $\mathbf{x}$. The overall expression is prone to bias, since the approximation would be based on the initial wave $f$. Therefore, the initial wave is replaced with the mean f-wave $\overline{\mathbf{f}}_{p}$ from the set of pure waves.

$$
J_{i}=\left\|\overline{\mathbf{f}}_{p}-\left(\mathbf{f}_{o, i}-\mathbf{M} \mathbf{b}_{i}\right)\right\|^{2}
$$


Optimizing the expression means finding the parameter b that minimizes the error, which in this case would be the coefficients of the polynomial that estimates the $\mathrm{T}$ wave. It is possible to do this thanks to the use of the $\ell_{2}$-norm. The optimized parameter is given as

$$
\mathbf{b}=\left(\mathbf{M}^{\mathrm{T}} \mathbf{M}\right)^{-1} \mathbf{M}^{\mathrm{T}}\left(\mathbf{f}_{o, i}-\overline{\mathbf{f}}_{p}\right)
$$

The polynomial coefficients were calculated and the resulting curve was subtracted from the observation. The maximum polynomial degree $K$ plays a role in determining the quality of estimation. Since no method exists to determine the optimal degree, it was set arbitrarily. In order to prevent modelization of the fine dynamics that are attributed to AFL, $K$ was limited to a low degree. In this paper, $K=3$. This results in f-wave estimates free from their local VA influence but still retaining the fine dynamics of AFL.

The recovered f-waves were split into two sets: pure corrected and overlapped corrected, noted as $\mathcal{F}_{p c}$ and $\mathcal{F}_{o c}$ respectively. These waves are defined only in the interval of their onsets and ends, thus introducing gaps between two waves in the reconstructed time series.

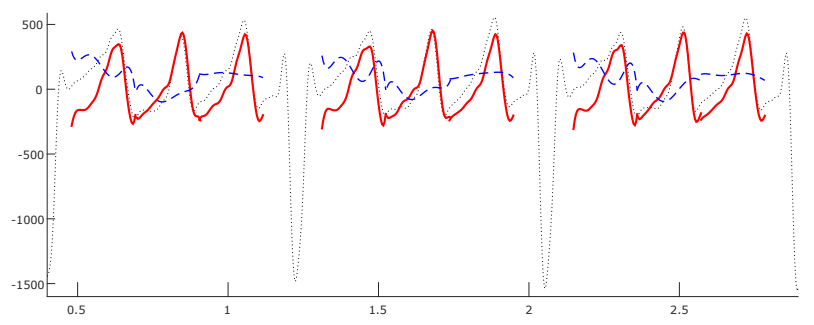

Figure 2. Corrected f-waves from (Fig 1) for lead V1. The red curves represent corrected f-waves, with their estimated local VA in blue dashed lines. Note the edge effect of the waves preceeding a QRS complex.

There exists an edge effect, as can be seen in (Fig 2) attributed to the use of the polynomial basis. To correct this, all f-waves were truncated at the onset and end to discard this effect and to also prevent wave overlap.

\subsection{Calculation of loop parameters}

Analysis of the post-processed data involves characterising the mean waveform from each set. This allows us to evaluate the steady-state nature of the AA and the changes that LSPE brings to it. In this paper, the vectorcardiographic (VCG) loop parameters such as orientation and geometry were analyzed, inspired by the authors of [3] and [4].

Each f-wave was tranformed into its VCG representation using the inverse Dower transform [5]. The spatial orientation of the VCG axes, made up of the 3 Frank leads
( $\mathrm{X}, \mathrm{Y}$ and $\mathrm{Z}$ ), is coherent with the axes of the body ( $\mathrm{X}$ indicating right and left, $\mathrm{Y}$ indicating superior and inferior, $\mathrm{Z}$ indicating anterior and posterior). The loop orientation and form was determined using the eigenvectors and eigenvalues issued from principal component analysis (PCA).

First, the mean loop of each set was calculated. Each loop was then downsampled to $100 \mathrm{~Hz}$ to keep only the pertinent information regarding AFL. To avoid bias between unevenly distributed points in spatial representation, each loop was resampled by interpolating points at its minimum Euclidean distance. PCA was then applied in order to obtain the eigenvectors $\mathbf{v}_{i}=\left[\begin{array}{lll}v_{i x} & v_{i y} & v_{i z}\end{array}\right]^{\mathrm{T}}$ and eigenvalues $\lambda_{i}, i \in[1 ; 3]$.

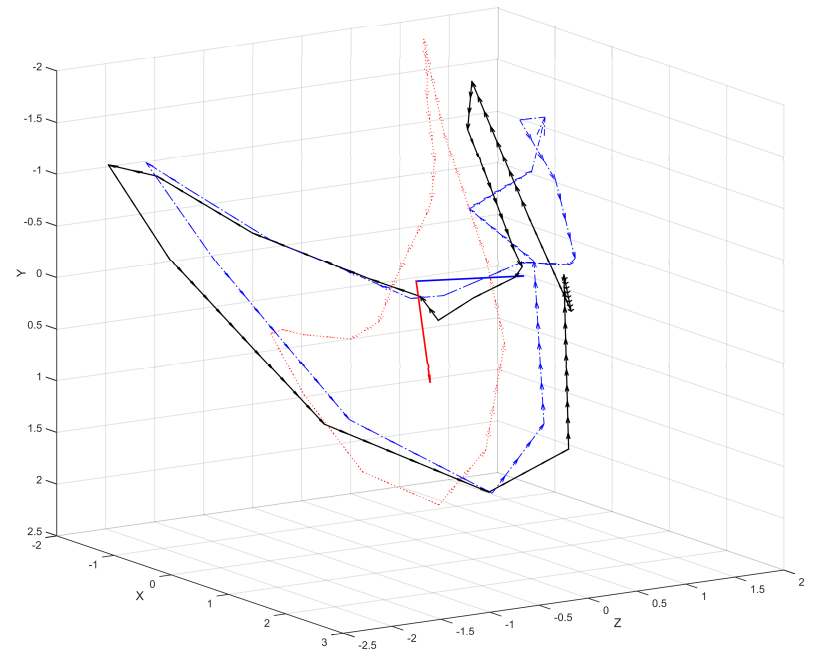

Figure 3. VCG loops of patient \#9. The black, dashed blue and dotted red loops correspond to the mean loop for sets $\mathcal{F}_{p}, \mathcal{F}_{o c}$ and $\mathcal{F}_{o}$ respectively. The red (pointing down) and blue (pointing right) vectors are the first and second eigenvectors of the mean pure loops.

Loop orientation, given in terms of azimuth $\phi_{A Z}$ and elevation $\phi_{E L}$ was calculated by finding the angle of intersection between the plane spanned by the first two eigenvectors $\mathbf{v}_{1}$ and $\mathbf{v}_{2}$ with planes $\mathrm{XZ}$ and $\mathrm{YZ}$ respectively.

$$
\begin{aligned}
& \phi_{A Z}=\arctan \left(\frac{v_{2 z}-\left(v_{2 y} / v_{1 y}\right) v_{1 z}}{v_{2 x}-\left(v_{2 y} / v_{1 y}\right) v_{1 x}}\right) \\
& \phi_{E L}=\arctan \left(\frac{v_{2 z}-\left(v_{2 x} / v_{1 x}\right) v_{1 z}}{v_{2 y}-\left(v_{2 x} / v_{1 x}\right) v_{1 y}}\right)
\end{aligned}
$$

Loop geometry, given in terms of planarity $\psi_{P L}$ and plane geometry (the shape of the loop) $\psi_{P G}$ was calculated using the eigenvalues.

$$
\begin{array}{r}
\psi_{P L}=\frac{\lambda_{3}}{\sum_{i=1}^{3} \lambda_{i}} \\
\psi_{P G}=\frac{\lambda_{2}}{\lambda_{1}}
\end{array}
$$




\subsection{Performance analysis of LSPE}

Performance quantification was done by evaluating the effect of LSPE on the parameters described previously. To do this, the distance error with respect to the pure wave parameter is calculated

$$
\epsilon_{\theta, a, b}=\left|\theta_{a}-\theta_{p}\right|-\left|\theta_{b}-\theta_{p}\right|
$$

where $\theta$ indicates the considered parameter, and $a$ and $b$ the two sets in comparison.

Table 1. Distance errors $\epsilon$.

\begin{tabular}{lcc}
\hline \hline Parameter & $\epsilon_{o, o c}$ & $\epsilon_{o c, p c}$ \\
\hline$\phi_{A Z}$ & $\mathbf{2 4 . 7 9 2} \pm \mathbf{6 . 7 9 6}$ & $\mathbf{3 . 6 0 0} \pm \mathbf{3 . 4 6 4}$ \\
$\phi_{E L}$ & $\mathbf{3 2 . 9 7 8} \pm \mathbf{5 . 5 5 5}$ & $\mathbf{3 . 3 2 8} \pm \mathbf{4 . 7 9 4}$ \\
$\psi_{P L}$ & $\mathbf{0 . 0 0 5} \pm \mathbf{0 . 0 0 4}$ & $0.001 \pm 0.003$ \\
$\psi_{P G}$ & $\mathbf{0 . 1 0 4} \pm \mathbf{0 . 0 3 7}$ & $\mathbf{0 . 0 2 3} \pm \mathbf{0 . 0 0 7}$ \\
\hline \hline
\end{tabular}

To quantify the corrective performance, the error was calculated between the mean loop parameters of sets $\mathcal{F}_{o}$ and $\mathcal{F}_{o c}$. A good corrective performance would give a larger first term than the second, hence giving a positive error value.

The corrective effect of LSPE was also evaluated. The error was calculated between the mean loop parameters of sets $\mathcal{F}_{o c}$ and $\mathcal{F}_{p c}$. Minimal corrective effect would give a smaller second term than the first, hence giving a positive error value.

Due to non-unique PCA eigenvector direction, the orientation may become flipped. Therefore, when calculating the error for $\phi_{A Z}$ and $\phi_{E L}$, the value that minimizes the distance (either $\theta$ or $\theta \bmod ( \pm \pi)$ ) was taken.

The error parameter $\epsilon$ was evaluated for all 25 records. Each parameter was tested for Gaussianity. In the negative, the rank-sum test was used to evaluate the median of the ensemble. It is expected to be as positive as possible. The results of both analysis are given in Table 1, presented as the median and standard deviation. A p-value $<0.05$ is considered statistically significant and is marked in bold.

\section{Results and Discussion}

There is net positivity in both distance errors for 3 of 4 parameters. It is shown from the first comparison that using corrected f-waves previously overlapped in $\mathrm{T}$ waves, we obtain significant improvement in parameter estimation. This is also visible in (Fig 3), where the mean loop from $\mathcal{F}_{o c}$ is very similar to the one from $\mathcal{F}_{p}$. The results also show that there is similarity between $\mathcal{F}_{p c}$ waves and $\mathcal{F}_{p}$ waves. This shows that LSPE, although applied onto pure f-waves, does not significantly affect the loop parameters.
The next step is to quantify the improvement in the classification of AFL non-invasively. As suggested by [3], the plane orientation may indicate a right typical AFL in the case of $\phi_{A Z} \approx-45 \mathrm{deg}$.

There are no known rules for determining the degree of polynomials $K$. There is a need to define an optimum value since it determines the quality of correction. In the case of maintaining a constant $K$, introduction of penalising weights according to the position of the f-wave in an RR interval might give a similar effect to an optimal $K$.

The detection of f-waves is key in applying LSPE. Better detection may be obtained using a generalized likelihood ratio test approach, taking into account the presence of VA in order to obtain a good test statistic.

\section{Conclusion}

Using LSPE, we can successfully recover f-waves that were superposed on top of T waves, giving better VCG loop parameter statistics than using pure f-waves alone. LSPE allows correction of T-wave component in $\mathrm{f}$-waves that are superposed, whilst minimally affecting pure fwaves. These f-waves may then be used to localize the ectopic source non-invasively.

\section{References}

[1] Barbato G, Carinci V, Tomasi C, Frassineti V, Margheri M, Pasquale GD. Is electrocardiography a reliable tool for identifying patients with isthmus-dependent atrial flutter? Europace 2009;1071-1076.

[2] Meo M, Zarzoso V, Meste O, Latcu DG, Saoudi N. Spatial variability of the 12-lead surface ecg as a tool for noninvasive prediction of catheter ablation outcome in persistent atrial fibrillation. IEEE Transactions on Biomedical Engineering Jan 2013;60(1):20-27. ISSN 0018-9294.

[3] Ng J, Sahakian AV, Fisher WG, Swiryn S. Atrial flutter vector loops derived from the surface ECG: does the plane of the loop correspond anatomically to the macroreentrant circuit? J Electrocardiol 2003;36:181-186. ISSN 00220736.

[4] Richter U, Stridh M, Bollmann A, Husser D, Sörnmo L. Spatial characteristics of atrial fibrillation electrocardiograms. J Electrocardiol 2008;41:165-172. ISSN 00220736.

[5] Dower GE, Machado HB, Osborne JA. On deriving the electrocardiogram from vectoradiographic leads. Clin Cardiol apr 1980;3(2):87-95. ISSN 0160-9289 (Print).

Address for correspondence:

Muhammad Haziq Kamarul Azman

Bureau 206

Laboratoire I3S

2000 route des Lucioles

06900 Sophia Antipolis, France

mhaziq@unikl.edu.my 